\title{
Factors associated with length of hospital stay among dialysis patients with nontraumatic acute abdomen: a retrospective observational study
}

\author{
Chang-Han $\underline{\mathrm{LO}}^{1}$, MD, Yu-Juei $\underline{\mathrm{Hs}}^{1,2}, \mathrm{MD}, \mathrm{PhD}$, Shun-Neng $\underline{\mathrm{Hsu}}^{1}$, MD, Chin $\underline{\mathrm{Lin}}^{3,4}$, PhD, Sui-Lung $\underline{\mathrm{Su}}^{3,4}$, PhD
}

INTRODUCTION Nontraumatic acute abdomen (NTAA) in dialysis patients is a challenging issue. The aetiologies of NTAA vary considerably depending on the renal replacement therapy (RRT) modality. Although haematological parameters and contributing factors have been reported to be associated with outcomes for dialysis patients, their clinical effect on the length of hospital stay (LOS) remains unknown.

METHODS We retrospectively analysed 52 dialysis patients (peritoneal dialysis [PD], $n=33$; haemodialysis [HD], $n=19$ ) and 30 non-dialysis patients (as controls) between January 2011 and December 2014. To attenuate the selection bias, non-dialysis patients with NTAA were matched to cases at a ratio of $1: 1$ by age, gender and comorbidities (diabetes mellitus and hypertension). Their demographic characteristics, laboratory data, clinical assessment scores and LOS were analysed. RESULTS The PD group exhibited a significantly higher neutrophil percentage, neutrophil-to-lymphocyte ratio (NLR), platelet-to-lymphocyte ratio (PLR); longer LOS; and lower lymphocyte percentage and absolute lymphocyte count than the control group. After multivariate analysis adjustment, female gender, longer RRT duration and higher intact parathyroid hormone (iPTH) levels were associated with a lower probability of being discharged home. In the dialysis group, a higher $\mathrm{iPTH}$ level ( $>313 \mathrm{pg} / \mathrm{mL}$ ) was positively correlated with longer LOS. iPTH level combined with NLR can be used as a surrogate marker for predicting longer LOS $(p<0.001)$.

CONCLUSION NTAA dialysis patients with female gender, longer RRT duration and higher iPTH levels are prone to experiencing longer LOS. In addition, the combination of IPTH and NLR is a significant determinant for LOS in NTAA dialysis patients.

Keywords: dialysis, intact parathyroid hormone, neutrophil-to-lymphocyte ratio, nontraumatic acute abdomen, platelet-to-lymphocyte ratio

\section{INTRODUCTION}

Gastrointestinal (GI) disorders are commonly encountered in both the general population and dialysis patients. Previous studies have reported a higher prevalence of GI disorders, such as gastritis and peptic ulcer disease (PUD), in end-stage renal disease (ESRD) patients than in their corresponding non-ESRD controls. ${ }^{(1-3)}$ Moreover, a retrospective cohort study showed a higher incidence of acute cholecystitis in ESRD patients (5.8 per 1,000 patient-years) than in their matched controls $(0.92$ per 1,000 patient-years); in addition, haemodialysis (HD) is an independent risk factor for acute cholecystitis. ${ }^{(4)}$ Patients on maintenance peritoneal dialysis (PD) are at an increased risk of acute pancreatitis (AP), ${ }^{(5-7)}$ which is associated with high morbidity and mortality and is difficult to diagnose early because its presentation is similar to that of PD-related peritonitis (PDRP).

The reported incidence of $\mathrm{Gl}$ symptoms, which ranges from $32 \%$ to $85 \%$, is variable in dialysis patients. ${ }^{(8-10)}$ Dong et al reported a significantly higher prevalence of Gl symptoms in HD patients than in PD patients (76.4\% vs. $61.6 \%) .{ }^{(11)}$ Conversely, a cross-sectional study that enrolled 294 dialysis patients found that $85 \%$ of the PD patients reported at least one Gl symptom compared with $51 \%$ of those on HD. ${ }^{(10)}$ Acute abdominal pain is the most common GI manifestation in dialysis patients and can be caused by a wide spectrum of GI disorders, ranging from mild indigestion to catastrophic mesenteric ischaemia. However, dialysis patients with abdominal catastrophes usually present with nonclassical symptoms, and results of their physical examinations can be misleadingly benign. Therefore, early and correct diagnosis of nontraumatic acute abdomen (NTAA) in dialysis patients is crucial and can significantly influence the outcome. Currently, there is limited research evaluating the applications of clinical parameters for early diagnosis and outcome prediction of GI disorders in dialysis patients. ${ }^{(8,9)}$

Many studies have identified numerous novel biomarkers for differentiating between the causes of acute abdomen, including high-sensitivity C-reactive protein, procalcitonin, smooth muscle myosin, D-dimers, soluble elastin fragments, fatty acidbinding protein, plasma citrulline and D-lactate. ${ }^{(12-14)}$ However, estimation of most of the aforementioned parameters may be time-consuming and may not be immediately available in the emergency department. A complete blood count is a simple and cheap routine test that can provide helpful clues to predict inflammation in dialysis patients. A recent study showed that HD patients with higher inflammation levels exhibited a higher neutrophil-to-lymphocyte ratio (NLR) and platelet-to-lymphocyte ratio (PLR), and that NLR and PLR were positively correlated with

${ }^{1}$ Division of Nephrology, Department of Internal Medicine, Tri-Service General Hospital, ${ }^{2}$ Graduate Institute of Medical Science, ${ }^{3}$ Graduate Institute of Life Sciences, ${ }^{4}$ School of Public Health, National Defense Medical Center, Taipei, Taiwan

Correspondence: Dr Chang-Han Lo, Visiting Staff, Division of Nephrology, Department of Internal Medicine, Tri-Service General Hospital, National Defense Medical Center, Taiwan, 325, Sec 2, Cheng-Kung Road, Neihu 114, Taipei, Taiwan ROC. twoworldwar66@gmail.com 
high-sensitivity C-reactive protein levels. ${ }^{(15)}$ Another cross-sectional study demonstrated that ESRD patients with higher PLR had significantly higher NLR, interleukin-6 (IL-6) and tumour necrosis factor- $\alpha$ (TNF- $\alpha$ ) levels than those without, suggesting that PLR predicts inflammation in ESRD patients. ${ }^{(16)}$ However, data on the impact of haematological parameters on early detection of NTAA in dialysis patients remains unavailable. Therefore, the aim of this study was to investigate the association between haematological parameters and NTAA, and to identify the risk factors associated with longer hospital length of stay (LOS) in dialysis patients.

\section{METHODS}

This study was reviewed and approved by the Institutional Review Board of the Tri-Service General Hospital, National Defense Medical Center, Taiwan (IRB No. 1-104-05-146). As this was a retrospective study in a dry lab context, informed consent was not necessary.

We retrospectively reviewed the medical records of selected $\mathrm{HD}, \mathrm{PD}$ and non-dialysis patients who were admitted through the emergency department of an academic medical centre (TriService General Hospital) because of NTAA from January 2011 to December 2014. For the dialysis group, we excluded (a) patients who were pregnant or had a traumatic history before the acute abdomen event; (b) those aged $<20$ years or $>90$ years; and those who underwent renal replacement therapy (RRT) for $<3$ months. Non-dialysis patients who were hospitalised because of NTAA within 30 days of entry were recruited as the control group and matched to dialysis cases by age, gender and comorbidities (diabetes mellitus and hypertension).

The following data was retrieved from the medical records of all eligible subjects: age, gender, primary renal disease, RRT duration and modality, time to diagnosis, prior abdominal surgery, respiratory rate (RR), heart rate, body temperature (BT), systolic blood pressure, diastolic blood pressure, nutrition status, comorbidity, and LOS. We also collected the following laboratory data: white blood cell count (WBC), haemoglobin ( $\mathrm{Hb}$ ) level, platelet count, neutrophil percentage, lymphocyte percentage, NLR, absolute lymphocyte count (ALC), PLR, and C-reactive protein (CRP), serum sodium, creatinine, urea, albumin, intact parathyroid hormone (iPTH) and total bilirubin (TB) levels. These were measured using standard laboratory techniques on an automatic analyser (AU 5000 chemistry analyser; Olympus, Tokyo, Japan). Sequential organ failure assessment (SOFA), ${ }^{(17)}$ systemic inflammatory response syndrome $(\mathrm{SIRS})^{(18)}$ and bedside index of severity in AP (BISAP $)^{(19,20)}$ scores were also determined as previously described.

A diagnosis of PDRP was made based on the presence of (a) cloudy effluent, (b) an effluent cell count $\geq 100$ cells/ $\mu \mathrm{L}$, and (c) $\geq 50 \%$ polymorphonuclear cells in the differential cell count. ${ }^{(21)}$ Complete blood counts with automated differential counts, which included total white blood cells, neutrophils and lymphocytes, were obtained at the time of admission. NLR and PLR were calculated.

All variables were expressed as mean \pm standard deviation or numbers and percentages. Continuous variables were evaluated
Table I. Final diagnosis of acute abdomen among the peritoneal dialysis (PD), haemodialysis (HD) and control groups.

\begin{tabular}{|c|c|}
\hline Variable & No. (\%) \\
\hline \multicolumn{2}{|l|}{ PD $(n=33)$} \\
\hline PD-related peritonitis & $24(72.7)$ \\
\hline Acute pancreatitis & $4(12.1)$ \\
\hline Herpes zoster & $2(6.1)$ \\
\hline Peptic ulcer disease & $1(3.0)$ \\
\hline Intestinal obstruction & $1(3.0)$ \\
\hline Exit-site infection & $1(3.0)$ \\
\hline \multicolumn{2}{|l|}{ HD (n = 19) } \\
\hline Intestinal obstruction & $6(31.6)$ \\
\hline Biliary tract infection & $4(21.1)$ \\
\hline Acute pancreatitis & $1(5.3)$ \\
\hline Herpes zoster & $1(5.3)$ \\
\hline Peptic ulcer disease & $1(5.3)$ \\
\hline Small bowel angiodysplasia & $1(5.3)$ \\
\hline Diverticulitis & $1(5.3)$ \\
\hline Hepatocellular carcinoma & $1(5.3)$ \\
\hline Acute appendicitis & $1(5.3)$ \\
\hline Renal cyst complications & $1(5.3)$ \\
\hline Portal vein thrombophlebitis & $1(5.3)$ \\
\hline \multicolumn{2}{|l|}{ Control $(n=30)$} \\
\hline Intestinal obstruction & $9(30.0)$ \\
\hline Biliary tract infection & $6(20.0)$ \\
\hline Acute gastroenteritis & $5(16.7)$ \\
\hline Acute pancreatitis & $4(13.3)$ \\
\hline Peptic ulcer disease & $2(6.7)$ \\
\hline Constipation & $1(3.3)$ \\
\hline Irritable bowel syndrome & $1(3.3)$ \\
\hline Intra-papillary mucinous neoplasm & $1(3.3)$ \\
\hline Ketamine abuse & $1(3.3)$ \\
\hline
\end{tabular}

using Student's t-test, Wilcoxon rank-sum test, and Kruskal-Wallis test. Categorical variables were evaluated using chi-square test and Fisher's exact test. A Cox proportional hazard model was used to estimate the crude hazard ratios (HRs) and adjusted HRs (aHRs) of gender, RRT durations, iPTH level and SIRS. 95\% confidence intervals (Cls) were used to evaluate the association between risk factors and the probability of being discharged home. We used a conditional inference tree to identify the most important variables that could predict LOS in dialysis patients. A p-value $<0.05$ was considered significant for all analyses. Statistical analyses were conducted using R software version 3.2.3 (R Project for Statistical Computing, Vienna, Austria).

\section{RESULTS}

A total of 52 dialysis patients with NTAA (33 on PD, 19 on HD) were enrolled. The control group consisted of 30 non-dialysis patients admitted because of NTAA. The aetiologies of NTAA in the dialysis and control groups are depicted in Table I.

As shown in Table II, no significant differences were observed in gender, mean age, blood pressure and BT between the PD and 
Table II. Clinical characteristics of the peritoneal dialysis (PD), haemodialysis (HD) and control groups.

\begin{tabular}{|c|c|c|c|c|c|}
\hline \multirow[t]{2}{*}{ Variable } & \multicolumn{3}{|c|}{ Mean \pm standard deviation/no. (\%) } & \multirow{2}{*}{$\begin{array}{l}\text { p-value } \\
\text { (PD vs. control) }\end{array}$} & \multirow{2}{*}{$\begin{array}{l}\text { p-value } \\
\text { (HD vs. control }\end{array}$} \\
\hline & PD $(n=33)$ & HD (n = 19) & Control $(n=30)$ & & \\
\hline Gender & & & & 0.942 & 0.176 \\
\hline Male & $14(42.42)$ & $12(63.16)$ & $13(43.33)$ & & \\
\hline Female & $19(57.58)$ & $7(36.84)$ & $17(56.67)$ & & \\
\hline Age (yr) & $54.00 \pm 12.68$ & $64.63 \pm 16.84$ & $56.67 \pm 22.13$ & 0.555 & 0.222 \\
\hline Prior IA surgery & & & & 0.035 & 0.063 \\
\hline No & $24(72.73)$ & $14(73.68)$ & $14(46.67)$ & & \\
\hline Yes & $9(27.27)$ & $5(26.32)$ & $16(53.33)$ & & \\
\hline Respiratory rate (times/min) & $18.85 \pm 0.87$ & $19.21 \pm 1.78$ & $17.83 \pm 0.83$ & $<0.001$ & $<0.001$ \\
\hline Heart rate (beats/min) & $92.42 \pm 17.54$ & $82.32 \pm 8.61$ & $80.47 \pm 13.08$ & 0.003 & 0.517 \\
\hline SBP (mmHg) & $136.94 \pm 26.06$ & $133.74 \pm 30.59$ & $128.10 \pm 17.52$ & 0.123 & 0.466 \\
\hline Body temperature $\left({ }^{\circ} \mathrm{C}\right)$ & $37.06 \pm 0.91$ & $36.98 \pm 0.71$ & $36.90 \pm 0.62$ & 0.421 & 0.503 \\
\hline WBC $\left(\times 10^{3} / \mathrm{mm}^{3}\right)$ & $11,550.91 \pm 5,357.84$ & $10,283.16 \pm 4,194.04$ & $10,692.00 \pm 4,800.83$ & 0.507 & 0.975 \\
\hline Haemoglobin (g/dL) & $10.12 \pm 1.99$ & $10.57 \pm 2.09$ & $13.41 \pm 2.07$ & $<0.001$ & $<0.001$ \\
\hline Platelet count $\left(10^{3} / \mu \mathrm{L}\right)$ & $243.67 \pm 82.52$ & $180.47 \pm 65.53$ & $232.73 \pm 74.62$ & 0.585 & 0.035 \\
\hline Neutrophil (\%) & $82.24 \pm 7.90$ & $74.86 \pm 11.64$ & $72.66 \pm 14.00$ & 0.001 & 0.743 \\
\hline Lymphocyte (\%) & $10.55 \pm 4.89$ & $16.85 \pm 9.08$ & $20.06 \pm 12.46$ & $<0.001$ & 0.505 \\
\hline $\operatorname{ALC}\left(\times 10^{3} / \mathrm{mm}^{3}\right)$ & $1,090.10 \pm 558.23$ & $1,521.89 \pm 721.59$ & $1,786.31 \pm 884.86$ & $<0.001$ & 0.354 \\
\hline NLR & $10.97 \pm 8.77$ & $6.73 \pm 5.53$ & $6.46 \pm 6.46$ & 0.025 & 0.633 \\
\hline PLR & $270.96 \pm 145.11$ & $137.42 \pm 64.91$ & $158.59 \pm 90.92$ & 0.001 & 0.576 \\
\hline Amylase (U/L) & $106.60 \pm 124.08$ & $178.67 \pm 182.05$ & $192.90 \pm 500.61$ & 0.762 & 0.022 \\
\hline Lipase (U/L) & $383.71 \pm 722.01$ & $965.17 \pm 1,874.34$ & $98.08 \pm 233.87$ & $<0.001$ & 0.012 \\
\hline C-reactive protein (mg/L) & $7.05 \pm 8.17$ & $10.14 \pm 11.89$ & $7.57 \pm 10.02$ & 0.832 & 0.158 \\
\hline Albumin (mg/dl) & $3.26 \pm 0.55$ & $3.49 \pm 0.63$ & $3.57 \pm 0.58$ & 0.044 & 0.775 \\
\hline Time to diagnosis (day) & $2.67 \pm 2.29$ & $4.89 \pm 6.91$ & $4.73 \pm 6.64$ & 0.097 & 0.468 \\
\hline Hospital LOS (day) & $14.18 \pm 23.90$ & $12.47 \pm 15.73$ & $5.23 \pm 2.54$ & 0.046 & 0.034 \\
\hline RRT vintage (mth) & $51.58 \pm 30.74$ & $49.63 \pm 23.40$ & & & \\
\hline Creatinine (mg/dL) & $11.04 \pm 2.94$ & $6.66 \pm 2.91$ & $0.90 \pm 0.24$ & $<0.001$ & $<0.001$ \\
\hline Blood urea nitrogen (mg/dL) & $65.27 \pm 23.16$ & $48.89 \pm 25.83$ & $13.35 \pm 7.38$ & $<0.001$ & $<0.001$ \\
\hline Total bilirubin (mg/dL) & $0.52 \pm 0.38$ & $0.71 \pm 0.84$ & $1.53 \pm 1.73$ & 0.002 & 0.002 \\
\hline SOFA & $4.27 \pm 0.80$ & $4.05 \pm 0.85$ & $0.80 \pm 1.13$ & $<0.001$ & $<0.001$ \\
\hline SIRS & $1.12 \pm 0.86$ & $0.84 \pm 0.83$ & $0.63 \pm 0.93$ & 0.034 & 0.229 \\
\hline BISAP & $2.15 \pm 0.91$ & $1.84 \pm 0.96$ & $0.83 \pm 0.79$ & $<0.001$ & $<0.001$ \\
\hline
\end{tabular}

ALC: absolute lymphocyte count; BISAP: bedside index of severity in acute pancreatitis; DBP: diastolic blood pressure; IA: intra-abdominal; LOS: length of stay; NLR: neutrophil-to-lymphocyte ratio; PLR: platelet-to-lymphocyte ratio; RRT: renal replacement therapy; SBP: systolic blood pressure; SIRS: systemic inflammatory response syndrome; SOFA: sequential organ failure assessment; WBC: white blood cell count

control groups. However, a significant difference was observed in the prior intra-abdominal surgery ratio, RR and heart rate between the two groups. The haematological variables of neutrophil percentage, NLR and PLR were significantly higher in the PD group. Compared with the control group, the PD group exhibited significantly lower lymphocyte percentage, ALC, and levels of $\mathrm{Hb}$, serum sodium and serum albumin, but significantly higher blood urea nitrogen, creatinine and lipase levels. The PD group also had significantly higher SOFA, SIRS and BISAP scores as well as longer LOS.

There were no significant differences between the HD and control groups with respect to gender, age, prior intra-abdominal surgery ratio, heart rate, blood pressure and BT. A significant difference was only observed in RR between the HD and control groups. The haematological variables of neutrophil percentage, lymphocyte percentage, NLR, ALC and PLR were comparable between the HD and control groups. The HD group exhibited significantly lower platelet counts, Hb levels and TB levels and significantly higher blood urea nitrogen and creatinine levels compared to the control group. However, serum sodium and albumin levels were similar. The HD group also had significantly higher SOFA and BISAP scores and longer LOS.

PDRP can be diagnosed on the basis of abdominal pain together with a cloudy effluent, whereas the clues for early diagnosis of non-PDRP-related NTAA are not easily recognised in dialysis patients. To find out the useful parameters for differential 
Table III. Clinical characteristics of the PDRP group and non-PDRP group in dialysis patients.

\begin{tabular}{|c|c|c|c|}
\hline \multirow[t]{2}{*}{ Variable } & \multicolumn{2}{|c|}{ Mean \pm standard deviation/No. (\%) } & \multirow[t]{2}{*}{ p-value } \\
\hline & PDRP $(n=24)$ & Non-PDRP $(n=28)$ & \\
\hline Age (yr) & $53.54 \pm 14.07$ & $61.61 \pm 15.18$ & 0.054 \\
\hline Gender & & & 0.587 \\
\hline Male & $13(54.2)$ & $13(46.4)$ & \\
\hline Female & $11(45.8)$ & $15(53.6)$ & \\
\hline Prior abdominal surgery & & & 0.209 \\
\hline Yes & $11(45.8)$ & $8(28.6)$ & \\
\hline No & $13(54.2)$ & $20(71.4)$ & \\
\hline Respiratory rate (times/min) & $18.83 \pm 0.86$ & $19.11 \pm 1.54$ & 0.446 \\
\hline Heart rate (beats/min) & $92.42 \pm 19.53$ & $85.57 \pm 10.58$ & 0.134 \\
\hline BT ( $\left.{ }^{\circ} \mathrm{C}\right)$ & $37.26 \pm 0.96$ & $36.80 \pm 0.63$ & 0.046 \\
\hline SBP (mmHg) & $137.00 \pm 27.85$ & $134.71 \pm 27.74$ & 0.769 \\
\hline $\mathrm{DBP}(\mathrm{mmHg})$ & $78.13 \pm 10.67$ & $76.71 \pm 14.04$ & 0.689 \\
\hline WBC $\left(\times 10^{3} / \mathrm{mm}^{3}\right)$ & $11.59 \pm 5.74$ & $10.65 \pm 4.23$ & 0.498 \\
\hline Haemoglobin (g/dL) & $9.97 \pm 2.19$ & $10.55 \pm 1.85$ & 0.309 \\
\hline Platelet count $\left(\times 10^{3} / \mathrm{mm}^{3}\right)$ & $233.71 \pm 77.02$ & $209.32 \pm 85.90$ & 0.290 \\
\hline Neutrophil (\%) & $84.32 \pm 6.81$ & $75.44 \pm 10.55$ & 0.001 \\
\hline Lymphocyte (\%) & $9.50 \pm 4.57$ & $15.72 \pm 8.04$ & 0.001 \\
\hline $\operatorname{ALC}\left(\times 10^{3} / \mathrm{mm}^{3}\right)$ & $0.97 \pm 0.56$ & $1.48 \pm 0.64$ & 0.004 \\
\hline NLR & $12.44 \pm 9.57$ & $6.83 \pm 5.14$ & 0.015 \\
\hline PLR & $298.96 \pm 159.48$ & $156.34 \pm 65.77$ & $<0.001$ \\
\hline Ferritin (mg/dL) & $528.88 \pm 1,061.24$ & $606.29 \pm 863.06$ & 0.778 \\
\hline C-reactive protein $(\mathrm{mg} / \mathrm{dL})$ & $8.33 \pm 8.65$ & $7.87 \pm 10.66$ & 0.870 \\
\hline Total calcium (mg/dL) & $9.24 \pm 0.90$ & $9.28 \pm 0.93$ & 0.873 \\
\hline Phosphate (mg/dL) & $5.04 \pm 1.31$ & $4.44 \pm 1.70$ & 0.171 \\
\hline iPTH (pg/mL) & $285.37 \pm 238.69$ & $385.8 \pm 416.82$ & 0.306 \\
\hline Sodium (mg/dL) & $131.5 \pm 3.63$ & $135.75 \pm 3.69$ & $<0.001$ \\
\hline Albumin (mg/dL) & $3.19 \pm 0.55$ & $3.49 \pm 0.59$ & 0.068 \\
\hline Lipase (mg/dL) & $62.00 \pm 44.8$ & $920.25 \pm 1,572.67$ & 0.029 \\
\hline Total bilirubin (mg/dL) & $0.53 \pm 0.43$ & $0.64 \pm 0.69$ & 0.522 \\
\hline SOFA & $4.25 \pm 0.84$ & $4.14 \pm 0.80$ & 0.642 \\
\hline SIRS & $1.17 \pm 0.86$ & $0.89 \pm 0.83$ & 0.252 \\
\hline BISAP & $2.08 \pm 0.83$ & $2.00 \pm 1.01$ & 0.750 \\
\hline Time to diagnosis (day) & $2.29 \pm 1.65$ & $4.50 \pm 5.96$ & 0.070 \\
\hline Hospital LOS (day) & $11.08 \pm 11.30$ & $15.68 \pm 26.91$ & 0.440 \\
\hline
\end{tabular}

ALC: absolute lymphocyte count; BISAP: bedside index of severity in acute pancreatitis; BT: body temperature; DBP: diastolic blood pressure; HR: heart rate; iPTH: intact parathyroid hormone; LOS: length of stay; NLR: neutrophil-to-lymphocyte ratio; PDRP: peritoneal dialysis-related peritonitis; PLR: platelet-to-lymphocyte ratio; SBP: systolic blood pressure; SIRS: systemic inflammatory response syndrome; SOFA: sequential organ failure assessment; WBC: white blood cell count

diagnosis of NTAA, we compared the clinical manifestations between PDRP and non-PDRP-related NTAA in dialysis patients. As shown in Table III, we did not observe a significant difference in gender, prior abdominal surgery ratio, $\mathrm{RR}$, heart rate, blood pressure, $\mathrm{WBC}, \mathrm{Hb}$, platelet count, ferritin, CRP, total calcium, phosphate, iPTH or TB between the PDRP and non-PDRPrelated NTAA group. In addition, the PDRP group also showed a significantly higher $B T$, neutrophil percentage, NLR and PLR, and a significantly lower lymphocyte percentage, ALC, serum sodium and lipase than the non-PDRP group. PDRP patients were also slightly younger and had a shorter time to diagnosis than those with non-PDRP NTAA. However, SOFA, SIRS and BISAP scores were comparable between the two groups.
A multivariate Cox proportional hazard regression analysis was used to estimate crude HRs, aHRs and $95 \% \mathrm{Cls}$ for determining the probability of being discharged in the dialysis group with NTAA. We observed that female gender (aHR 0.412, $p=0.011$ ), longer RRT duration (aHR 0.980, $p=0.001$ ) and higher iPTH levels (aHR 0.998, $p=0.001$ ) were significantly associated with a lower probability of being discharged, after adjustment for gender, RRT duration, iPTH level and SIRS (Table IV). Additionally, a higher SIRS score was associated with a lower probability of being discharged, although this was not significant (aHR 0.675, $\mathrm{p}=0.052$ ).

To identify the most important predictors of LOS in dialysis patients with NTAA, we built a conditional inference tree to 
Table IV. Probability of being discharged home for dialysis patients.

\begin{tabular}{|lllll|}
\hline Independent variable & Crude HR (95\% Cl) & p-value & aHR* (95\% Cl) & p-value \\
\hline Female gender & $0.538(0.341-0.848)$ & 0.008 & $0.412(0.208-0.815)$ & 0.011 \\
\hline RRT duration $(\mathrm{mth})$ & $0.986(0.976-0.996)$ & 0.009 & $0.980(0.969-0.992)$ & 0.001 \\
\hline iPTH & $0.999(0.998-1.000)$ & 0.006 & $0.998(0.997-0.999)$ & 0.001 \\
\hline SIRS & $0.655(0.497-0.864)$ & 0.003 & $0.675(0.454-1.003)$ & 0.052 \\
\hline
\end{tabular}

*All aHR results were adjusted for gender, RRT duration, iPTH and SIRS. Cl: confidence interval; HR: hazard ratio; iPTH: intact parathyroid hormone; RRT: renal replacement therapy; SIRS: systemic inflammatory response syndrome

estimate the regression relationship between some variables of interest identified in the aforementioned analyses and LOS by using binary recursive partitioning. The results of the conditional inference tree are shown in Fig. 1. The decision tree analysis identified serum iPTH level as the first split: dialysis patients with higher iPTH levels (> $313 \mathrm{pg} / \mathrm{mL}$ ) had longer LOS than those with lower iPTH levels $(\leq 313 \mathrm{pg} / \mathrm{mL})$. Within the dialysis group with lower iPTH levels $(\leq 313 \mathrm{pg} / \mathrm{mL})$, we identified NLR to be a second split. Compared with lower NLR values ( $\leq 10.294)$, higher NLR values (> 10.294) were associated with longer LOS in the dialysis group that had lower iPTH levels $(\leq 313 \mathrm{pg} / \mathrm{mL})$. Thus, dialysis patients were divided into three groups based on iPTH and NLR: Group 1 - lower iPTH and lower NLR; Group 2 - lower iPTH and higher NLR; Group 3 - higher iPTH. Fig. 2 shows the Kaplan-Meier curve of the LOS of the three groups. The Cox models were also used to prove the superiority of the combination of these parameters $(p<0.001)$. The $r^{2}$ values of the models using iPTH (cut-off point $=313 \mathrm{pg} / \mathrm{mL}$ ) and NLR (cut-off point $=10.294$ ) were $14.1 \%$ and $1.3 \%$, respectively. In addition, this combination provided an $\mathrm{r}^{2}$ of $19.7 \%$ for predicting the probability of being discharged.

\section{DISCUSSION}

In this study, we demonstrated that intestinal obstruction (IO) and biliary tract infection (BTI) were the most two common causes of NTAA in dialysis patients on HD and non-dialysis patients, whereas PDRP was the most common cause of NTAA in dialysis patients on PD. Compared with the controls, PD patients had significantly altered haematological profiles, including neutrophilia, lymphopenia, increased PLR and NLR, and anaemia. In HD patients, thrombocytopenia was evident. Both HD and PD patients exhibited elevated lipase levels, and PD patients tended to have hyponatraemia. By contrast, female gender, longer RRT duration and higher iPTH levels were significant independent risk factors associated with a lower probability of being discharged home. The decision tree analysis showed that dialysis patients with lower iPTH $(\leq 313 \mathrm{pg} / \mathrm{mL})$ and NLR $(\leq 10.294)$ levels had the shortest LOS.

Although the symptoms of GI disorders are common among dialysis patients, a paucity of literature exists concerning the prevalence of NTAA in dialysis patients on HD or PD. Using the National Health Insurance Research Database of Taiwan, a recent large-scale retrospective cohort study enrolled 8,955 incident HD patients, 1,791 incident PD patients and a comparison cohort of 8,955 non-dialysis patients. It reported that the risks of gastro-oesophageal reflux disease, $\mathrm{IO}$ and abdominal hernia were significantly higher in the PD group, whereas the risks of PUD, lower GI diverticula and bleeding were significantly higher in the HD group. Furthermore, the risks of mesenteric ischaemia, liver cirrhosis and AP were higher in dialysis patients, but no significant difference was observed between the PD and HD groups. ${ }^{(22)}$ By contrast, the present study saw a higher prevalence of IO in the HD group than the PD group. This finding can be explained by the relatively high prevalence of prior surgery $(26.32 \%)$ in the HD group and the lack of reported events of adhesion secondary to PDRP in our PD group. Consistent with the results of another nationwide population-based cohort study, we demonstrated a higher prevalence of BTI in the HD group than the PD group. ${ }^{(4)}$ Although the mechanism underlying this observation has yet to be elucidated, fluctuating haemodynamic status during HD sessions leading to hypoperfusion and ischaemia of the gallbladder may be one of the contributors.

ESRD, a well-established immunodeficient state, has detrimental effects on both innate and adaptive immune cells. Lymphopenia is the clinical manifestation of a defective adaptive immune system in patients with ESRD; loss of renal function is associated with reduced numbers of circulating $T$ cells and $B$ cells, leading to significant T- and B-cell lymphopenia. Compared with $T$ cells from healthy subjects, the cells isolated from ESRD patients exhibit an increased susceptibility to activationinduced apoptosis. In addition, the low thymic output of naive $\mathrm{T}$ cells may explain T-cell lymphopenia in ESRD patients. ${ }^{(23)}$ Impaired responsiveness to B-cell activating factor and increased activation-induced B-cell apoptosis may also contribute to B-cell lymphopenia. ${ }^{(23)}$ Together, impaired functions and the increased apoptosis of both $\mathrm{T}$ and $\mathrm{B}$ cells may result in an increased risk of infection, impaired vaccination responses and reduced tumour surveillance, leading to an increased risk of cancer in patients with ESRD. Consistently, we observed that ESRD patients on PD or HD had lymphopenia, and infectious insults such as PDRP, exit-site infection, pancreatitis, BTI, diverticulitis and acute appendicitis.

It has been established that dialysis patients exhibit increased serum levels of inflammatory mediators including IL-1 (IL-1 $\beta$ ), IL-6, and TNF- $\alpha$. However, cytokines measurement is costly and time-consuming; thus, cytokines are not applicable surrogate indexes of inflammatory status for dialysis patients in clinical practice. In addition to leucopenia, we also demonstrated that NLR and PLR, which are inexpensive and readily available indicators, were significantly associated with NTAA in PD patients. Consistent with a previous study, we observed that elevated NLR and PLR levels were associated with NTAA in PD patients. PD patients with NTAA exhibited significant neutrophilia 


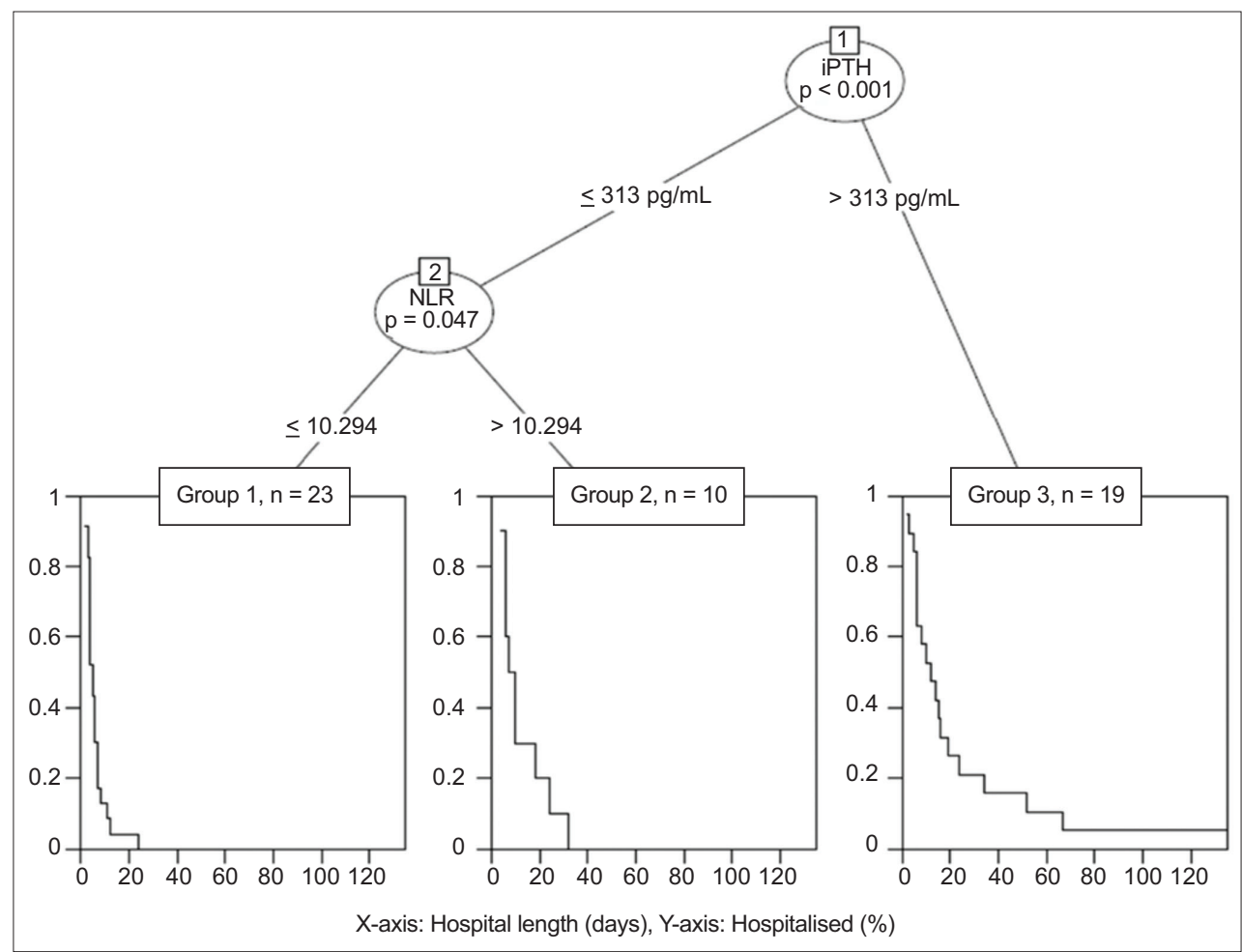

Fig. 1 Conditional inference tree shows a positive correlation between higher iPTH levels ( $>313 \mathrm{pg} / \mathrm{mL})$ and longer hospital length of stay ( $p<0.001)$, as well as lower iPTH $(\leq 313 \mathrm{pg} / \mathrm{mL})$ and higher NLR ( $>10.294)$ and longer hospital length of stay $(p=0.047)$. Dialysis patients were classified into three groups based on iPTH levels and NLR. Group 1: lower iPTH and lower NLR; Group 2: lower iPTH and higher NLR; Group 3: higher iPTH; iPTH: intact parathyroid hormone; NLR: neutrophil-to-lymphocyte ratio

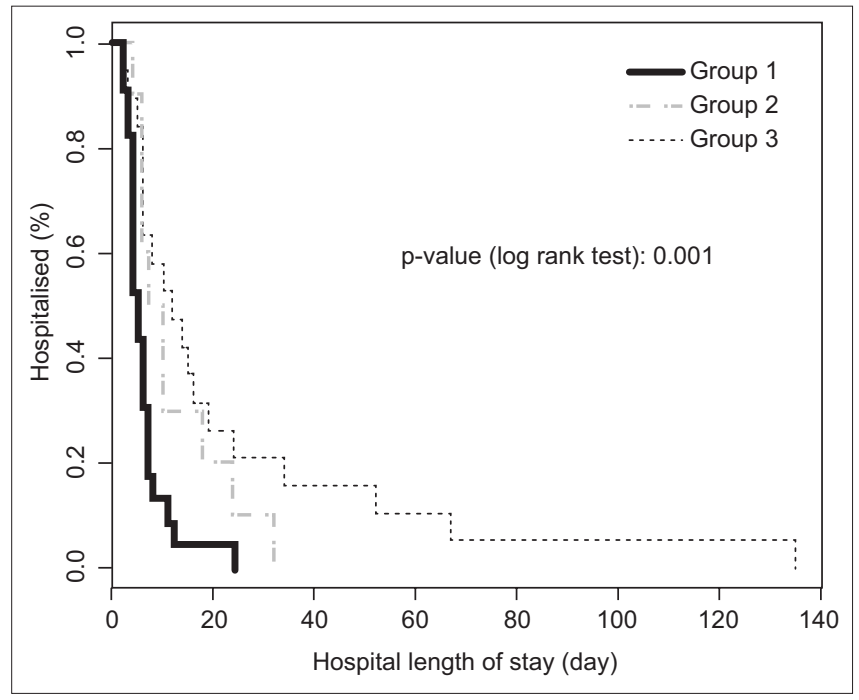

Fig. 2 Kaplan-Meier curve shows the probability of each group being discharged, with Group 1 having a higher probability of discharge than Group 2, followed by Group 3. Group 1: lower iPTH and lower NLR; Group 2: lower iPTH and higher NLR; Group 3: higher iPTH; iPTH: intact parathyroid hormone; NLR: neutrophil-to-lymphocyte ratio

and lymphopenia, which may result in elevated NLR and PLR, as compared to controls with NTAA. By contrast, we found that HD patients with NTAA had significantly lower, rather than higher, PLR compared with that of PD patients and controls with NTAA. This discrepancy may be explained by relative thrombocytopenia in HD patients. Moreover, NLR and PLR have been shown to be associated with all-cause mortality in HD patients, ${ }^{(24)}$ and NLR can predict vascular calcification in ESRD patients. ${ }^{(25)}$ In this study, we further demonstrated that the combination of NLR and serum
iPTH can predict the probability of being discharged in dialysis patients with NTAA (Figs. 1 \& 2).

In this study, female gender was an independent risk factor associated with a lower probability of being discharged home. The reason for this is unclear. Similarly, Tomino et al demonstrated high mortality in female patients compared with male patients $(1 / 1$ [100\%] vs. $3 / 8$ [37.5\%]) in terms of emergency surgery for acute abdomen in dialysis patients. ${ }^{(26)}$ We provide two explanations for this phenomenon. First, NTAA arising from gynaecological disease only occurs in female patients. Hence, they may require a longer hospital LOS for the correct diagnosis to be made. Second, oestrogen in the fertile female patient offers a protective effect, including the regulation of particular metabolic factors such as lipids, the coagulant system and inflammatory markers, that expires after menopause, ${ }^{(27)}$ like in the female patients in our study. However, a prospective study needs to be conducted to confirm the gender discrepancy.

Secondary hyperparathyroidism is a universal finding in ESRD patients on HD or PD. In this study, using binary recursive partitioning, we demonstrated that dialysis patients with NTAA who had lower iPTH levels ( $\leq 313 \mathrm{pg} / \mathrm{mL}$ ) exhibited shorter LOS than those who had higher iPTH levels (> $313 \mathrm{pg} / \mathrm{mL}$ ). Although the reasons that dialysis patients with lower iPTH levels had a shorter LOS for NTAA remain unclear, previous studies have observed that patients with primary hyperparathyroidism (PHPT) may present with some digestive disorders, mostly abdominal pain and constipation. ${ }^{(28)}$ Hypercalcaemia, increased gastric acid secretion and the sustained stimulation of parathyroid hormone receptors located on the GI tracts may explain the development of 
digestive symptoms in PHPT patients, and significant resolution of these symptoms is observed after parathyroidectomy. Moreover, PHPT has been proven to be an aetiology of AP, with the reported incidence of AP in patients with PHPT varying from $1.5 \%$ to $15.3 \%$. $^{(29)}$ Jacob et al also reported a 28 -fold higher risk of pancreatitis in patients with PHPT than in the general population. ${ }^{(30)}$ Hypercalcaemia, rather than PHPT, seems to be more related to the development of pancreatitis; excess $\mathrm{Ca}^{2+}$ ion deposition within the pancreatic ducts may lead to consequent obstruction and inflammation. By contrast, Felderbauer et al demonstrated that genetic risk factors including serine protease inhibitor Kazal-type I and cystic fibrosis transmembrane conductance regulator gene mutations, are associated with the occurrence of pancreatitis in PHPT patients. ${ }^{(31)}$ The relationship between PTH and Gl disorders requires further exploration.

Hyponatraemia, a common laboratory finding, is associated with increased morbidity and mortality in patients with congestive heart failure, coronary artery disease, cirrhosis and cancer. In HD patients, hyponatraemia is associated with malnutrition, inflammation, fluid overload and mineral metabolism, and also predicts all-cause mortality. ${ }^{(32)}$ Similarly, lower serum sodium levels at the beginning of RRT are independently associated with a higher risk of death. Kim et al also demonstrated a clear association between hyponatraemia and new-onset cardiovascular events in PD patients. ${ }^{(33)}$ In this study, we found that the average serum sodium levels were significantly lower in the PD group than the control group. Consistent with our observations, previous studies have reported that HD patients with appendicitis who had hyponatraemia exhibited a longer LOS and higher morbidity than those without hyponatraemia. ${ }^{(34)}$ Moreover, hyponatraemia is associated with a high incidence of Gram-negative bacilli infection and malnutrition, long LOS and a high mortality rate in PD patients with PDRP. ${ }^{(35)}$ In contrast to patients with normal renal function, inadequate fluid removal, reduced salt intake because of poor appetite, and water shift from the intracellular space to the extracellular space secondary to accelerated degradation of intracellular osmoles may be involved in the development of hyponatraemia in dialysis patients with NTAA.

SIRS, SOFA and BISAP are common scoring systems for assessing the outcome of patients with sepsis, critical illness and AP. However, limited studies have compared the performance of these scoring systems in ESRD patients. In this study, the SOFA and BISAP scoring systems did not have superior discriminative power and a stronger association with patient outcome compared with the conventional SIRS scoring system. In dialysis patients, severe azotaemia may lessen the discriminative abilities of the SOFA and BISAP scoring systems, which originally contain renal function parameters.

This study has several limitations. First, as it was retrospective and not a randomised control trial, bias could have occurred. Second, the study was limited to a relatively small sample of dialysis patients with NTAA, hence its prognostic accuracy and selection bias must be carefully evaluated. Third, there were possible differences in the prevalence and outcomes of dialysis patients with NTAA among races and countries. Fourth, detailed information regarding patients' current use of medications, such as analgesic agents that can mask abdominal pain and influence patient enrolment, was not available in our study.

In summary, we demonstrated that female gender, longer RRT duration and higher iPTH levels were independent risk factors associated with a lower probability of being discharged home. In addition, dialysis patients with lower iPTH $(\leq 313 \mathrm{pg} / \mathrm{mL})$ and NLR $(\leq 10.294)$ levels had the shortest LOS. NLR and iPTH can be utilised as potential markers for predicting LOS in dialysis patients with NTAA. Since these conclusions are based on a small retrospective cohort, further prospective studies would be needed to confirm these findings.

\section{REFERENCES}

1. Cano AE, Neil AK, Kang JY, et al. Gastrointestinal symptoms in patients with end-stage renal disease undergoing treatment by hemodialysis or peritoneal dialysis. Am J Gastroenterol 2007; 102:1990-7.

2. Murtagh FE, Addington-Hall J, Higginson IJ. The prevalence of symptoms in endstage renal disease: a systematic review. Adv Chronic Kidney Dis 2007; 14:82-99.

3. Liang $\mathrm{CC}$, Muo $\mathrm{CH}$, Wang IK, et al. Peptic ulcer disease risk in chronic kidney disease: ten-year incidence, ulcer location, and ulcerogenic effect of medications. PLoS One 2014; 9:e87952.

4. Chen YT, Ou SM, Chao PW, et al. Acute cholecystitis in end-stage renal disease patients: a nation-wide longitudinal study. Dig Liver Dis 2013; 45:142-6.

5. Quraishi ER, Goel S, Gupta M, et al. Acute pancreatitis in patients on chronic peritoneal dialysis: an increased risk? Am J Gastroenterol 2005; 100:2288-93.

6. Bruno MJ, van Westerloo DJ, van Dorp WT, et al. Acute pancreatitis in peritoneal dialysis and haemodialysis: risk, clinical course, outcome, and possible aetiology. Gut 2000; 46:385-9.

7. Lankisch PG, Weber-Dany B, Maisonneuve P, Lowenfels AB. Frequency and severity of acute pancreatitis in chronic dialysis patients. Nephrol Dial Transplant 2008; 23:1401-5.

8. Strid H, Simrén M, Johansson AC, et al. The prevalence of gastrointestinal symptoms in patients with chronic renal failure is increased and associated with impaired psychological general well-being. Nephrol Dial Transplant 2002; 17:1434-9.

9. Dong R, Guo ZY. Gastrointestinal symptoms in patients undergoing peritoneal dialysis: multivariate analysis of correlated factors. World J Gastroenterol 2010; 16:2812-7

10. Salamon K, Woods J, Paul E, Huggins C. Peritoneal dialysis patients have higher prevalence of gastrointestinal symptoms than hemodialysis patients. J Ren Nutr 2013; 23:114-8.

11. Dong R, Guo ZY, Ding JR, Zhou YY, Wu H. Gastrointestinal symptoms: a comparison between patients undergoing peritoneal dialysis and hemodialysis. World J Gastroenterol 2014; 20:11370-5.

12. Kwan KY, Nager AL. Diagnosing pediatric appendicitis: usefulness of laboratory markers. Am J Emerg Med 2010; 28:1009-15.

13. Ranasinghe AM, Bonser RS. Biomarkers in acute aortic dissection and other aortic syndromes. J Am Coll Cardiol 2010; 56:1535-41.

14. Piton G, Capellier G. Biomarkers of gut barrier failure in the ICU. Curr Opin Crit Care 2016; 22:152-60.

15. Ahbap E, Sakaci T, Kara E, et al. Neutrophil-to-lymphocyte ratio and plateletto-lymphocyte ratio in evaluation of inflammation in end-stage renal disease. Clin Nephrol 2016; 85:199-208.

16. Balta S, Demırkol S, Kucuk U. The platelet lymphocyte ratio may be useful inflammatory indicator in clinical practice. Hemodial Int 2013; 17:668-9.

17. Ferreira FL, Bota DP, Bross A, Mélot C, Vincent JL. Serial evaluation of the SOFA score to predict outcome in critically ill patients. JAMA 2001; 286:1754-8.

18. Bone RC. Toward an epidemiology and natural history of SIRS (systemic inflammatory response syndrome). JAMA 1992; 268:3452-5.

19. Gao W, Yang HX, Ma CE. The value of BISAP score for predicting mortality and severity in acute pancreatitis: a systematic review and meta-analysis. PLoS One 2015; 10:e0130412.

20. Qiu L, Sun RQ, Jia RR, et al. Comparison of existing clinical scoring systems in predicting severity and prognoses of hyperlipidemic acute pancreatitis in Chinese patients: a retrospective study. Medicine (Baltimore) 2015; 94:e957.

21. Lane JC, Warady BA, Feneberg R, et al; International Pediatric Peritonitis Registry. Relapsing peritonitis in children who undergo chronic peritoneal dialysis: a prospective study of the international pediatric peritonitis registry. Clin J Am Soc Nephrol 2010; 5:1041-6.

22. Lee YC, Hung SY, Wang HH, et al. Different risk of common gastrointestinal disease between groups undergoing hemodialysis or peritoneal dialysis or with non-end stage renal disease: a nationwide population-based cohort study. Medicine (Baltimore) 2015; 94:e1482. 
23. Meijers RW, Betjes MG, Baan CC, Litjens NH. T-cell ageing in end-stage renal disease patients: assessment and clinical relevance. World J Nephrol 2014; 3:268-76.

24. Yaprak M, Turan MN, Dayanan R, et al. Platelet-to-lymphocyte ratio predicts mortality better than neutrophil-to-lymphocyte ratio in hemodialysis patients. Int Urol Nephrol 2016; 48:1343-8.

25. Turkmen K, Ozcicek F, Ozcicek A, et al. The relationship between neutrophil-tolymphocyte ratio and vascular calcification in end-stage renal disease patients. Hemodial Int 2014; 18:47-53.

26. Tomino T, Uchiyama $\mathrm{H}$, Itoh $\mathrm{S}$, et al. Outcomes of emergency surgery for acute abdomen in dialysis patients: experience of a single community hospital. Surg Today $2014 ; 44: 690-5$

27. Maas AH, Appelman YE. Gender differences in coronary heart disease. Neth Heart J 2010; 18:598-602.

28. Misgar RA, Dar PM, Masoodi SR, et al. Clinical and laboratory profile of primary hyperparathyroidism in Kashmir Valley: a single-center experience. Indian J Endocrinol Metab 2016; 20:696-701.
29. Bai HX, Giefer M, Patel M, Orabi Al, Husain SZ. The association of primary hyperparathyroidism with pancreatitis. J Clin Gastroenterol 2012; 46:656-61. 30. Jacob JJ, John M, Thomas N, et al. Does hyperparathyroidism cause pancreatitis? A South Indian experience and a review of published work. ANZ J Surg 2006; 76:740-4.

31. Felderbauer $\mathrm{P}$, Karakas $\mathrm{E}$, Fendrich $\mathrm{V}$, et al. Pancreatitis risk in primary hyperparathyroidism: relation to mutations in the SPINK1 trypsin inhibitor (N34S) and the cystic fibrosis gene. Am J Gastroenterol 2008; 103:368-74.

32. Zhang R, Wang S, Zhang M, Cui L. Hyponatremia in patients with chronic kidney disease. Hemodial Int 2017; 21:3-10.

33. Kim HW, Ryu GW, Park CH, et al. Hyponatremia predicts new-onset cardiovascular events in peritoneal dialysis patients. PLoS One 2015; 10:e0129480.

34. Kim DY, Nassiri N, de Virgilio C, et al. Association between hyponatremia and complicated appendicitis. JAMA Surg 2015; 150:911-2.

35. Tseng MH, Cheng CJ, Sung CC, et al. Hyponatremia is a surrogate marker of poor outcome in peritoneal dialysis-related peritonitis. BMC Nephrol 2014; 15:113. 